P. Mantica, C. Angioni, B. Baiocchi, M. Baruzzo, M.N.A. Beurskens, J.P.S. Bizarro, R.V. Budny, P. Buratti, A. Casati, C. Challis, J. Citrin, G. Colyer, F. Crisanti, A.C.A. Figueiredo, L. Frassinetti, C. Giroud, N. Hawkes, J. Hobirk, E. Joffrin, T. Johnson, E. Lerche, P. Migliano, V. Naulin, A.G. Peeters, G. Rewoldt, F. Ryter, A. Salmi, R. Sartori, C. Sozzi, G. Staebler, D. Strintzi, T. Tala, M. Tsalas, D. Van Eester, T. Versloot, P.C. deVries, J. Weiland and JET EFDA contributors

\title{
Ion Heat Transport Studies in JET
}




\section{Ion Heat Transport Studies in JET}

P. Mantica ${ }^{1}$, C. Angioni $^{2}$, B. Baiocchi ${ }^{1,3}$, M. Baruzzo ${ }^{4}$, M.N.A. Beurskens ${ }^{5}$, J.P.S. Bizarro ${ }^{6}$, R.V. Budny ${ }^{7}$, P. Buratti ${ }^{8}$, A. Casati ${ }^{9}$, C. Challis ${ }^{5}$, J. Citrin ${ }^{10}$, G. Colyer ${ }^{5}$, F. Crisanti ${ }^{8}$, A.C.A. Figueiredo ${ }^{6}$, L. Frassinetti ${ }^{11}$, C. Giroud ${ }^{5}$, N. Hawkes ${ }^{5}$, J. Hobirk ${ }^{2}$, E. Joffrin ${ }^{9}$, T. Johnson ${ }^{11}$, E. Lerche ${ }^{12}$, P. Migliano ${ }^{1,13}$, V. Naulin ${ }^{14}$, A.G. Peeters ${ }^{15}$, G. Rewoldt ${ }^{2}$, F. Ryter ${ }^{2}$, A. Salmi ${ }^{16}$, R. Sartori ${ }^{17}$, C. Sozzi ${ }^{1}$, G. Staebler ${ }^{18}$, D. Strintzi ${ }^{2}$, T. Tala ${ }^{19}$, M. Tsalas ${ }^{10}$, D. Van Eester ${ }^{12}$, T. Versloot ${ }^{10}$, P.C. deVries ${ }^{10}$, J. Weiland $^{20}$ and JET EFDA contributors*

\section{JET-EFDA, Culham Science Centre, OX14 3DB, Abingdon, UK}

\footnotetext{
${ }^{I}$ Istituto di Fisica del Plasma 'P.Caldirola', Associazione Euratom-ENEA-CNR, Milano, Italy

${ }^{2}$ Max-Planck-Institut für Plasmaphysik, EURATOM Association, Garching, Germany

${ }^{3}$ Università degli Studi di Milano, Dept. of Physics, Milano, Italy

${ }^{4}$ Consorzio RFX, ENEA-Euratom Association, Padua, Italy
}

${ }^{5}$ EURATOM-CCFE Fusion Association, Culham Science Centre, OX14 3DB, Abingdon, OXON, UK

${ }^{6}$ Associação EURATOM-IST, Instituto de Plasmas e Fusão Nuclear, Instituto Superior Técnico, Lisboa, Portugal

${ }^{7}$ Princeton Plasma Physics Laboratory, Princeton, New Jersey, 08543, USA

${ }^{8}$ Associazione EURATOM-ENEA sulla Fusione, C.R. Frascati, Frascati , Italy

${ }^{9}$ Association EURATOM-CEA, CEA/IRFM, F-13108 Saint Paul Lez Durance, France

${ }^{10}$ FOM Institute Rijnhuizen, Association EURATOM-FOM, Nieuwegein, the Netherlands

${ }^{11}$ Association EURATOM - VR, Fusion Plasma Physics, EES, KTH, Stockholm, Sweden

${ }^{12}$ LPP-ERM/KMS, Association EURATOM-Belgian State, TEC, B-1000 Brussels, Belgium

${ }^{13}$ Università degli Studi di Milano Bicocca, Dept. of Physics, Milano, Italy

${ }^{14}$ Association EURATOM-Risø DTU, DK-4000 Roskilde, Denmark

${ }^{15}$ University of Bayreuth, 95440 Bayreuth, Germany

${ }^{16}$ Association EURATOM-Tekes, Aalto University, Department of Applied Physics, Finland

${ }^{17}$ Fusion For Energy Joint Undertaking, Josep Pla 2, 08019, Barcelona, Spain

${ }^{18}$ General Atomics, P.O. Box 85608, San Diego, California 92186-5608, USA

${ }^{19}$ Association EURATOM-Tekes, VTT, P.O. Box 1000, FIN-02044 VTT, Finland

${ }^{20}$ Chalmers University of Technology and EURATOM-VR Association, Göteborg Sweden

* See annex of F. Romanelli et al, "Overview of JET Results",

(23rd IAEA Fusion Energy Conference, Daejon, Republic of Korea (2010)).

Preprint of Paper to be submitted for publication in Proceedings of the 38th EPS Conference on Plasma Physics

Strasbourg, France

(27th June 2011 - 1st July 2011) 
"This document is intended for publication in the open literature. It is made available on the understanding that it may not be further circulated and extracts or references may not be published prior to publication of the original when applicable, or without the consent of the Publications Officer, EFDA, Culham Science Centre, Abingdon, Oxon, OX14 3DB, UK."

"Enquiries about Copyright and reproduction should be addressed to the Publications Officer, EFDA, Culham Science Centre, Abingdon, Oxon, OX14 3DB, UK."

The contents of this preprint and all other JET EFDA Preprints and Conference Papers are available to view online free at www.iop.org/Jet. This site has full search facilities and e-mail alert options. The diagrams contained within the PDFs on this site are hyperlinked from the year 1996 onwards. 



\section{ABSTRACT.}

Detailed experimental studies of ion heat transport have been carried out in JET exploiting the upgrade of Active Charge Exchange Spectroscopy and the availability of multi-frequency ICRH with ${ }^{3} \mathrm{He}$ minority. The determination of ion threshold and stiffness offers unique opportunities for validation of the well-established theory of Ion Temperature Gradient driven modes. Ion stiffness is observed to decrease strongly in presence of toroidal rotation when the magnetic shear is sufficiently low. This effect is dominant with respect to the well-known $\omega_{\text {ExB }}$ threshold up-shift and plays a major role in enhancing core confinement in Hybrid regimes and Ion Internal Transport Barriers. The effects of $\mathrm{T}_{\mathrm{e}} / \mathrm{T}_{\mathrm{i}}$ and $\mathrm{s} / \mathrm{q}$ on ion threshold are found rather weak in the domain explored. Quasi-linear fluid/gyro-fluid and linear/non-linear gyro-kinetic simulations have been carried out. Whilst threshold predictions show good match with experimental observations, some significant discrepancies are found on the stiffness behaviour.

\section{INTRODUCTION}

Ion heat transport has been early addressed by extensive theoretical treatment [e.g.1-4], however the lack of well resolved ion temperature diagnostics has hindered until recently a precise experimental characterization and therefore a thorough theory validation.

The JET tokamak $(\mathrm{R}=2.96 \mathrm{~m}, \mathrm{a}=1 \mathrm{~m})$ [5] is equipped with high quality active Charge Exchange Spectroscopy $(\mathrm{CX})$ [6] for ion temperature $\left(\mathrm{T}_{\mathrm{i}}\right)$ and toroidal rotation $\left(\omega_{\mathrm{t}}\right)$ measurements and a multi-frequency Ion Cyclotron Resonance Heating (ICRH) for flexible and fairly localized ion heating using $\left({ }^{3} \mathrm{He}\right)-\mathrm{D}$ minority scheme [7]. These tools, together with JET's large size and low normalized ion gyro-radius, make it an ideal device to perform on ions studies of threshold and stiffness as earlier performed on electrons [8-9].

Ion Temperature Gradient (ITGs) modes feature a threshold in the inverse ion temperature gradient length $\left(\mathrm{R} / \mathrm{L}_{\mathrm{Ti}}=\mathrm{R} \mid \nabla \mathrm{T}_{\mathrm{i}} / \mathrm{T}_{\mathrm{i}}\right.$, with $\mathrm{R}$ the tokamak major radius) above which the ion heat flux $\left(\mathrm{q}_{\mathrm{i}}\right)$ increases strongly with $\mathrm{R} / \mathrm{L}_{\mathrm{Ti}}$. This property leads to stiffness of $\mathrm{T}_{\mathrm{i}}$ profiles with respect to changes in heating profiles. The level of stiffness characterizes how strongly $\mathrm{T}_{\mathrm{i}}$ profiles are tied to the threshold. The threshold value is theoretically predicted to decrease with increasing $\mathrm{T}_{\mathrm{e}} / \mathrm{T}_{\mathrm{i}}$ and with decreasing s/q [2,4], whilst the effect of rotation results mainly in a threshold up-shift according to the well-known "Waltz" quenching rule [10]. Ion stiffness is not often specifically addressed in theoretical literature, in particular no systematic investigation of the parametric dependence of ion stiffness is to our knowledge available. In cases where ion stiffness has been evaluated by nonlinear gyro-kinetic simulations, it is generally reported that ions are characterized by a rather high level of stiffness [e.g.11-13].

In this paper, we review the main results on ion threshold and stiffness obtained in JET in the last five years and the intense validation effort carried out by using quasi-linear fluid/gyro-fluid models and linear/non-linear gyro-kinetic simulations. Sect.2 describes the experimental set-up, Sect. 3 the role of rotational and magnetic shear, Sect.4 the role of $\mathrm{T}_{\mathrm{e}} / \mathrm{T}_{\mathrm{i}}$ and s/q, Sect.5 presents the conclusions and their implications and provides some indications for future work. 


\section{EXPERIMENTAL SET-UP}

Experimentally the identification of the ITG threshold and stiffness requires a scan of the core $\mathrm{q}_{\mathrm{i}}$ at constant edge $\mathrm{q}_{\mathrm{i}}$, to keep edge properties constant, whilst maintaining reasonably unchanged other plasma parameters. Heat fluxes are predicted by theory to follow a gyro-Bohm scaling, so that $\mathrm{q}_{\mathrm{i}}$ can be written in a general way as [14]

$$
q_{i}=q_{i}^{r e s}+n_{i} q^{1.5} \chi_{s} \frac{T_{i} \rho_{i}}{e B R^{2}} \frac{R}{L_{T_{i}}} f\left[\frac{R}{L_{T_{i}}}-\frac{R}{L_{T_{i c r i t}}}\right] \cdot H\left[\frac{R}{L_{T_{i}}}-\frac{R}{L_{T_{i c r i t}}}\right]
$$

where $\mathrm{q}_{\mathrm{i}}^{\text {res }}$ is the residual flux (basically the neoclassical flux), $\mathrm{n}_{\mathrm{i}}$ the ion density, $\mathrm{q}$ the safety factor, $B$ the magnetic field, e the electron charge, $\rho_{i}=\left(m_{i} T_{i}\right)^{1 / 2} / e B, m_{i}$ the ion mass and $H$ the Heaviside step function. Eq.(1) will be referred to in the following as Critical Gradient Model (CGM), R/ $\mathrm{L}_{\text {Ticrit }}$ as threshold and $\chi_{\mathrm{s}}$ as stiffness coefficient. From the curve of the gyro-Bohm normalized flux $\mathrm{q}_{\mathrm{i}}{ }^{\mathrm{GB}} \mathrm{vs} \mathrm{R} / \mathrm{L}_{\mathrm{Ti}}$, the threshold can be identified as the intercept at neoclassical flux and the stiffness coefficient can be inferred from the slope. To allow comparison with previous work on electrons, in this paper $\mathrm{f}\left(\mathrm{R} / \mathrm{L}_{\mathrm{Ti}}\right)$ is assumed linear, so $\mathrm{q}_{\mathrm{i}}$ is quadratic in $\mathrm{R} / \mathrm{L}_{\mathrm{Ti}}$.

The ion transport experiments were all performed in low triangularity JET L-mode plasmas with $\mathrm{B}_{\mathrm{T}}=3.36 \mathrm{~T}, \mathrm{I}_{\mathrm{p}}=1.8 \mathrm{MA}$ (with ramps up to $3 \mathrm{MA}$ for q profile manipulation), $\mathrm{n}_{\mathrm{e} 0} \sim 3-410^{19} \mathrm{~m}^{-3}$. The identification of threshold requires reaching very small core $\mathrm{q}_{\mathrm{i}}$, which can only be achieved with off-axis ICRH and no NBI (only blips of the CX beam). Therefore the threshold can only be measured in low rotation plasmas. Stiffness can instead be identified both at low and high rotation, either from the slope of the $\mathrm{q}_{i}{ }^{\mathrm{GB}}$ versus $\mathrm{R} / \mathrm{L}_{\mathrm{Ti}}$ curve or from $\mathrm{T}_{\mathrm{i}}$ modulation, as described in great detail in [13]. ICRH up to 7 MW (including ITER-like antenna) was in most cases applied in a resonant minority $\left({ }^{3} \mathrm{He}\right)$-D scheme $\left(33 \mathrm{MHz}\right.$ on-axis, $29 \mathrm{MHz}$ off-axis at $\left.\rho_{\text {tor }} \sim 0.6\right)$ with ${ }^{3} \mathrm{He}$ concentration $\sim 7 \%$ and $50-80 \%$ of the ICRH core power delivered to thermal ions. $(\mathrm{H})-\mathrm{D}$ and $\left({ }^{3} \mathrm{He}\right)-\mathrm{D}$ Mode Conversion ( ${ }^{3} \mathrm{He} \sim 15-20 \%$ ) were used as electron heating to increase the $\mathrm{T}_{\mathrm{e}} / \mathrm{T}_{\mathrm{i}}$ ratio. To obtain high rotation, NBI power up to $12 \mathrm{MW}$ was applied. Values of $\mathrm{R} / \mathrm{L}_{\mathrm{Ti}}$ from $\mathrm{CX}$ (time resolution: 10 $\mathrm{ms}$, space resolution: $8 \mathrm{~cm}$ ) were averaged in time over the stationary intervals and calculated with respect to the flux surface minor radius $\rho=\left(R_{\text {out }}-R_{\text {in }}\right) / 2$, where $R_{\text {out }}$ and $R_{\text {in }}$ are the outer and inner boundaries of the flux surface on the magnetic axis plane. The values of $q_{i}$ have been calculated using the SELFO [15] or PION [16] codes for ICRH and the PENCIL [17] code for NBI. Error bars are typically $\Delta \mathrm{R} / \mathrm{L}_{\mathrm{Ti}} \sim \pm 0.3-0.6$ and $\Delta \mathrm{q}_{\mathrm{i}}{ }^{\mathrm{GB}} \sim \pm 3$. The q profiles have been reconstructed by EFIT with pressure profile and MSE or polarimeter constraints. $\mathrm{n}_{\mathrm{e}}$ and $\mathrm{T}_{\mathrm{e}}$ were measured by LIDAR or High Resolution Thomson Scattering and $\mathrm{T}_{\mathrm{e}}$ also by ECE radiometer.

\section{EFFECT OF ROTATION AND Q PROFILE ON ION STIFFNESS 3.1 RESULTS OF TRANSPORT EXPERIMENTS}

Toroidal rotation emerges from JET experiments as the parameter that is mostly affecting the central part of the $T_{i}$ profiles [12]. Figures $1 \mathrm{a}, \mathrm{b}$ show the clear change in ion stiffness with rotation at $\rho_{\text {tor }}=0.33$, whilst at $\rho_{\text {tor }}=0.64$ ions appear very stiff irrespective of rotation. The reason for the 
different behaviour has been identified in the value of magnetic shear (s) [18]. Low values of $s$ $(<\sim 0.7)$ appear a necessary condition for ion stiffness reduction by rotation. Dedicated q profile scans with ion threshold and stiffness determination have been performed and the data at $\rho_{\text {tor }}=$ 0.33 are summarized in figure 1c. Low rotation data show high stiffness irrespective of the $s$ value, and keep tight to threshold, whilst at high rotation the stiffness reduction is larger at low s, allowing to reach $\mathrm{R} / \mathrm{L}_{\mathrm{Ti}}$ up to 10 even at low $\mathrm{q}_{\mathrm{i}}^{\mathrm{GB}}$. At $\rho_{\text {tor }}=0.64$ instead the stiffness level is always very high, with $\mathrm{R} / \mathrm{L}_{\mathrm{Ti}}$ values similar to those in figure1b. In figure $1 \mathrm{c}$ it is also shown that even higher $\mathrm{R} / \mathrm{L}_{\mathrm{Ti}}$ $(>10)$ is seen in high rotation shots when the $\mathrm{q}=2$ surface is located at low $\mathrm{s}$. This evidence is in line with observations of the beneficial role of low order rationals at small s on turbulent transport [19-22], for which a theoretical basis was proposed in [23]. The effect of rationals is reported both on ions and electrons [19-22] and also in absence of rotation [19, 22], so it appears as a different phenomenon, which adds to the stiffness mitigation discussed here, which is only observed on ions and strictly linked to rotation. The non-linear interaction between the two processes (through the link between ion heat and momentum transport) is likely the physics mechanism that allows reaching the highest $\mathrm{R} / \mathrm{L}_{\mathrm{Ti}}$ values, as discussed also in Sect.3.2.

The stiffness dependence on $s$ in presence of rotation gives origin to a common (but not really taken care of) feature in JET rotating shots, i.e. that $\mathrm{T}_{\mathrm{i}}$ profile peaking decreases in time with current diffusion (Figure 2a). This is actually a simple observation that could be made in other machines to check if the same physics is also at play. In low rotating shots this $\mathrm{T}_{\mathrm{i}}$ dynamics is completely absent (Figure $2 \mathrm{~b}$ ) and the $\mathrm{T}_{\mathrm{i}}$ peaking is always the lowest and actually slightly increasing in time, consistently with the expected dependence of the ITG threshold on s/q (cfr. Sect.3). Figure $3 a$ shows the variation of the $q$ and $n_{e}$ profiles between early and late times. A fairly small increase in density peaking is observed whilst the current peaks, which is anyway present in both low and high rotation shots and cannot account for the different $T_{i}$ behaviour. $T_{i}$ modulation data confirm the increase of ion stiffness as the q profile peaks in time in high rotation discharges. Figure $3 \mathrm{~b}$ shows profiles of the phase of the $\mathrm{T}_{\mathrm{i}}$ modulation at early and later times, indicating in the core region higher slopes (and therefore lower incremental diffusivity, i.e. lower stiffness [9]) at early times when the q profile is rather flat. The transport changes seen in figures $2 \mathrm{a}, \mathrm{b}$ are accompanied by consistent changes in turbulence measured by correlation reflectometry, as in figure $2 \mathrm{c}$. Two probing microwave beams are launched and the radial separation of their cut-off positions is scanned to obtain the reflectometer correlation length L [24]. Variations in L result from changes in the correlation length of turbulence but also in the turbulence amplitude [25]. Lower L values in the outer region are associated with higher turbulence amplitude. However, comparing data at the same radius, we see that lower $\mathrm{L}$ values are measured at a given time for the high rotation pulse, consistent with decreased turbulence correlation length in presence of larger rotational shear. In time, the decrease of core $\mathrm{R} / \mathrm{L}_{\mathrm{Ti}}$ shown in Fig.2a for the high rotation shot, and due to q profile peaking, is accompanied by a decrease of $\mathrm{L}$ at the inner radius, which, since rotational shear is stationary, can be attributed to rising core turbulence [25], associated with increased stiffness. At outer plasma positions where $\mathrm{R} / \mathrm{L}_{\mathrm{Ti}}$ keeps constant, also L remains constant. 
Finally, an experiment has been devised to discriminate whether the key ingredient for ion stiffness mitigation is the rotation value or its shear [26]. The key idea was to compare shots with standard $\mathrm{B}_{\mathrm{T}}$ ripple ( $\left.\sim 0.08 \%\right)$ and shots with enhanced $\mathrm{B}_{\mathrm{T}}$ ripple up to $1.5 \%$, in which both the edge and core rotation are reduced significantly by a counter-torque induced by fast ion losses, while in these discharges the core spatial gradient is less affected. Figure $4 \mathrm{a}$ shows that, in front of a significant change in rotation value without altering the gradient, the $T_{i}$ profile is unchanged. Figure $4 \mathrm{~b}$ summarizes all data. Low $\omega$ is $\omega<16 \mathrm{krad} / \mathrm{s}$, low (high) $\nabla \omega$ is $\nabla \omega<(>) 24 \mathrm{krad} / \mathrm{sm}$. It is clear that the high $\mathrm{B}_{\mathrm{T}}$ ripple data with low $\omega$ but high $\nabla \omega$ belongs to the class of shots with reduced stiffness, so we can draw the conclusion that it is the gradient of rotation that matters for stiffness mitigation, not the absolute value.

\subsection{EVIDENCE OF ION STIFFNESS MITIGATION IN JET ADVANCED TOKAMAK SCENARIOS}

The physics of ion stiffness reduction in presence of rotational shear and small s can be at the basis of all situations of core ion improved confinement, as observed in Hybrid and Internal Transport Barriers (ITB), providing an alternative paradigm to the usual interpretation in terms of ExB flow shear and threshold up-shift. In fact, all these regimes feature q profiles with broad regions of low $s$ and are always characterized by high rotation. In [27] the combined role of both rotation and low $s$ was already evidenced from JET ITB experimental data.

A sketch of the new proposed paradigm for the onset of improved ion core confinement is illustrated in figure 5. Full thick $\mathrm{T}_{\mathrm{i}}$ lines are the attainable profiles in presence of rotation, whilst dashed thin $\mathrm{T}_{\mathrm{i}}$ lines are the threshold profiles, to which $\mathrm{T}_{\mathrm{i}}$ is bounded in absence of rotation. The inner low s part in presence of rotation is dominated by stiffness effects, whilst the outer high s part (excluding the pedestal) is always stiff and close to threshold, which can increase slightly at higher $\mathrm{s} / \mathrm{q}$ or $\mathrm{T}_{\mathrm{i}} / \mathrm{T}_{\mathrm{e}}$. The non-stiff region is broadened by q profile shaping in Hybrids and ITBs with respect to $\mathrm{H}$-modes, so that the increased $\mathrm{R} / \mathrm{L}_{\mathrm{Ti}}$ due to rotation impacts also on global confinement. In ITBs in addition to stiffness mitigation an important effect is played by main rationals at low $\mathrm{s}$, which give an extra push in $\mathrm{R} / \mathrm{L}_{\mathrm{Ti}}$ (see also Sect.3.1), acting as a trigger of the onset of very large $\mathrm{R} / \mathrm{L}_{\mathrm{Ti}}$. This is due to the fact that the beneficial role of the rational increases the rotation gradient further, thereby further lowering ion stiffness in a non- linear feedback. The picture based on stiffness mitigation also implies that in absence of rotation no core ion improvement can take place, not even in presence of flat or reversed q profiles, which has been in fact experimentally confirmed both in JET [22, 29] and DIII-D [30]. In this case it was shown in [22] that, without the presence of de-stiffening by rotation, the rationals acting on stiff ions can only produce a glinch in $\mathrm{T}_{\mathrm{i}}$ but not a sustained ITB. In ITBs the enhancement in $\mathrm{H}_{98}$ factor to $\sim 1.5$ is completely due to the high core gradients, whilst in Hybrids it has been shown over a large database [28] that the contribution of pedestal to total energy is $20-40 \%$ (as in $\mathrm{H}$-modes) and the $\mathrm{H}_{98}$ factor improvement is due in equal parts to core and pedestal. The core improvement is found mainly located in the ion channel, which we interpret as due to stiffness mitigation, leading to an $\mathrm{H}_{98}$ increase by up to $\sim 0.2$. 
In order to find evidence in JET Hybrid and ITB plasmas that the dynamics of figure 5 are indeed at play, in figure 6a we examine their position in the $\mathrm{q}_{\mathrm{i}}^{\mathrm{GB}} \mathrm{vs} \mathrm{R} / \mathrm{L}_{\mathrm{Ti}}$ plot. The data at $\rho_{\text {tor }}=0.33$ populate uniformly the region of high $\mathrm{R} / \mathrm{L}_{\mathrm{Ti}}$ and low $\mathrm{q}_{\mathrm{i}}^{\mathrm{GB}}$. The problem here, in absence of $\mathrm{q}_{\mathrm{i}}$ scans or $\mathrm{T}_{\mathrm{i}}$ modulation, is to discriminate if this behaviour is mainly due to stiffness or threshold. In the core of Hybrids the linear threshold was found between 3.5 and 5 using GS2 [31], well below the actual $\mathrm{R} / \mathrm{L}_{\mathrm{Ti}}$. The flow shearing rate is $\sim 3-410^{4} \mathrm{~s}^{-1}$, yielding threshold up-shifts $\sim 1$. The profiles then lie well above threshold even at small $\mathrm{q}_{i}{ }^{\mathrm{GB}}$, indicating low stiffness. This was also confirmed by NBI $T_{i}$ modulation, which, although yielding lower and broader $T_{i}$ modulation than ICRH and with associated rotation modulation, still provides amplitude and phase profiles that can only be explained in presence of very low stiffness. Moreover, changing $\mathrm{T}_{\mathrm{i}}$ by degrading $\mathrm{T}_{\mathrm{i}}$ pedestal by increasing $\mathrm{B}_{\mathrm{T}}$ ripple allows a scan in $\mathrm{q}_{\mathrm{i}}{ }^{\mathrm{GB}}$, which also indicates low stiffness.

$\mathrm{R} / \mathrm{L}_{\mathrm{Ti}}$ vs $\mathrm{s}$ at low and high rotation is plotted in Figure $6 \mathrm{~b}$ from a JET H-mode/Hybrid database. The scatter is due to the range in parameters in the database, in first place $\mathrm{q}_{\mathrm{i}}$. Still, it is remarkable that the two clouds clearly separate at low s, with larger $\mathrm{R} / \mathrm{L}_{\mathrm{Ti}}$ at high rotation. The threshold values calculated using an analytical formula after [32] in the flat density limit

$$
R / L_{T_{i}}=\frac{4}{3}\left(1+\frac{T_{i}}{T_{e}}\right) \cdot\left(1+2 \frac{s}{q}\right) \quad \text { for } \quad \frac{R}{L_{n}}<2\left(1+\frac{T_{i}}{T_{e}}\right)
$$

are also plotted in figure $6 \mathrm{~b}$. They indicate again that low rotation data are kept near threshold by high stiffness, and they have the expected trend to increase with s/q, whilst the high rotation data are allowed to significantly depart from threshold at low $s$ by stiffness mitigation, but approach threshold again at high s, where rotation looses its effect on stiffness. This evidence suggests that stiffness mitigation in the broad low s region is at the origin of the improved core ion confinement in Hybrids. The dependence on $s$ is also one reason why not much effect is seen in fully-diffused $\mathrm{H}$-modes when ICRH power is substituted to NBI power, as discussed in [33]. In fact stiffness is observed to decrease from ICRH to NBI plasmas, but quantitatively the effect is weaker due to high s. In addition, ICRH has more peaked power deposition than NBI, so the local normalized flux value is higher and the two effects compensate, yielding similar $\mathrm{R} / \mathrm{L}_{\mathrm{Ti}}$. For ITBs, the profiles just before the trigger time yield $\omega_{\mathrm{ExB}} \sim 1-210^{4} \mathrm{~s}^{-1}$, not producing large threshold up-shift. Still, even in this phase the plasma is well above the linear threshold in presence of a heat flux well above neoclassical, which implies low stiffness. The ITB then develops, becoming particularly strong in presence of main rationals in low s regions, as discussed in [18]. In such fully developed ITB, the ITB itself generates a large localized rotation gradient at ITB location, with values of $\omega_{\mathrm{ExB}} \sim 7-810^{4} \mathrm{~s}^{-1}$, inducing significant threshold up-shifts. It is then difficult to separate the role of threshold and stiffness. Nevertheless, $T_{i}$ modulation has been performed using ${ }^{3} \mathrm{He}$ ICRH and shows that the ITB acts as a layer of very low incremental diffusivity, with sharp variation of amplitudes, indicating a very low slope of the $\mathrm{q}_{\mathrm{i}} \mathrm{vs}$ $\mathrm{R} / \mathrm{L}_{\mathrm{Ti}}$ plot, as discussed in detail in [18]. We conclude that also in ion ITBs the pattern of decreasing stiffness plays a major role, with the threshold up-shift intervening in a non-linear feedback whilst the ITB develops. 


\subsection{THEORETICAL INVESTIGATIONS}

In this section we address theory predictions of the effect of rotation on ion transport. In widely used quasi-linear transport models, such as Weiland [34] or GLF23 [35], which apply the Waltz rule on a given and restricted choice of spectral wave-numbers, rotation introduces only a (small) threshold up-shift and not a change in slope. Figure 7 a shows the results from the Weiland model for one discharge of Fig. 1 with $s=0.57$. The cases of no rotation and highest experimental rotation $\left(\gamma_{\mathrm{E}}=\omega_{\mathrm{ExB}} / \mathrm{c}_{\mathrm{s}} / \mathrm{a} \sim 0.1\right.$, with $\left.\mathrm{c}_{\mathrm{s}}=\left(\mathrm{T}_{\mathrm{e}} / \mathrm{m}_{\mathrm{i}}\right)^{1 / 2}\right)$ are compared, with clear disagreement with experiment. Instead, the more recent gyro-fluid TGLF model [36], which makes use of full spectra, indicates a change in slope, in particular in the region of the knee, where the transition between fully developed turbulence and zonal flows quenching takes place. Figure $7 \mathrm{~b}$ shows the TGLF simulations, starting from the parameters of one low rotation discharge and scanning $\mathrm{R} / \mathrm{L}_{\mathrm{Ti}}$ and $\mathrm{R} / \mathrm{L}_{\mathrm{Te}}$ in a prescribed ratio (taken from experiment) without and with rotation. Although the curve without rotation is not as steep as in experiment, we can clearly see a change in slope when adding rotation, which is actually dominant over the threshold up-shift, as in experiment. This effect is possibly due to the fact that there is differential suppression of turbulence at various wavelengths in the spectrum, with more suppression of the low stiffer wavelengths. Moreover, the change in stiffness with rotation is larger at low s, as shown in figure $7 \mathrm{~d}$ and in agreement with experiments. Following these findings, the Weiland model has recently been modified to include the dependence of the fastest growing mode number on rotation [37]. The results with such revised version are also shown in figure $7 \mathrm{a}$ and indicate a marked change in slope as in experiment. The impact of s on the stiffness change is also correctly reproduced (figure $7 \mathrm{e}$ ). The reason is that for large magnetic shear the radial correlation length is determined primarily by magnetic shear, whilst for low magnetic shear the correlation length is reduced by flow shear which cuts down larger eddies.

In order to verify these results, non-linear flux-tube electrostatic gyro-kinetic simulations using GYRO [38] have been made (figure 7c), including background rotation and electron-ion collisions, for the same parameters as with TGLF. The box size is $62 \times 222 \rho_{s}\left(\rho_{s}=\left(T_{e} m_{i}\right)^{1 / 2} / e B\right)$ in $x$ (radial) and $\mathrm{y}$ (binormal) directions, with 64 toroidal modes from $\mathrm{k}_{\mathrm{y}} \rho_{\mathrm{s}}=0.028$ to $\mathrm{k}_{\mathrm{y}} \rho_{\mathrm{s}}=1.78$ and with a minimum $\mathrm{k}_{\mathrm{x}} \rho_{\mathrm{s}}=0.1$ and $\max \mathrm{k}_{\mathrm{x}} \rho_{\mathrm{s}}=6.47$ corresponding to a radial resolution $\delta \mathrm{x} / \rho_{\mathrm{s}}=0.24$. The radial box size corresponds to $\Delta \mathrm{r} / \mathrm{a}=0.125$. Figure 8 illustrates the time behaviour of electrostatic potential comparing the $\mathrm{n}=0$ zonal component with the sum over all $\mathrm{n}>0$ components, for one simulation near marginality and for one simulation at high flux. Whilst at high values of $\mathrm{R} / \mathrm{L}_{\mathrm{Ti}}$ the simulations saturate quickly and even with a smaller number of toroidal modes yield the same flux, at low values of $\mathrm{R} / \mathrm{L}_{\mathrm{Ti}}$ development of very large values of $\mathrm{n}=0$ (zonal) electrostatic potential fluctuations is found, which strongly reduce transport and which obliged to perform simulations exceeding $1000 \mathrm{a} / \mathrm{c}_{\mathrm{s}}$. The same results are obtained with double radial box size keeping the same radial resolution. We note that near marginality runs with reduced number of toroidal modes are not delivering the same strength of zonal flows. With 64 modes, due to the large zonal flows, turbulent transport is rapidly quenched to zero near threshold and there is no hint of stiffness mitigation at high rotation, but basically only a threshold up-shift. Similar results were obtained also with GKW 
[39] as reported in [40] and recently with GS2 on a test case different from our experiments [41 Fig.3a]. Also non-linear fluid simulations of Resistive Ballooning Modes (RBM) turbulence, which has essential features common with ITG turbulence, have found no significant effect of rotation on ion stiffness [42]. Since the mechanisms that were mentioned above as possible origin of the stiffness reduction in quasi-linear models are also included, and with most resolved treatment, in non-linear gyro-kinetic models, we have to admit that presently the impact of rotation on ion stiffness remains an open issue on the theory side. Another open issue is that none of the models in figure 7 is in fact reproducing correctly the low $\mathrm{R} / \mathrm{L}_{\mathrm{Ti}}$ values of low rotation experimental data. The two quasi-linear models yield lower stiffness than experimental low rotation shots, whilst GYRO non-linear simulations yield higher $\mathrm{R} / \mathrm{L}_{\mathrm{Ti}}$ mainly due to the non-linear Dimits threshold up-shift, whilst the experimental low rotation $\mathrm{R} / \mathrm{L}_{\mathrm{Ti}}$ values stay close to the linear threshold $\mathrm{R} / \mathrm{L}_{\mathrm{Ti}, \text { crit }} \sim 3.6$. It has also been verified by global gyro-kinetic simulations that there is no effect of turbulence spreading inside $\rho_{\text {tor }}=0.4$. Future efforts should explore the issue with other available gyro-kinetic codes, comparing global versus flux-tube and also fixed-flux versus fixed-gradient simulations.

\section{PARAMETRIC DEPENDENCES OF ION THRESHOLD}

From the results in Sect.2 it is clear that effects of $\mathrm{T}_{\mathrm{e}} / \mathrm{T}_{\mathrm{i}}$ and $\mathrm{s} / \mathrm{q}$ on ion threshold should be investigated in low rotation plasmas, otherwise the phenomenon of stiffness mitigation due to rotation and low $s$ can dominate over the threshold dependences. This point however was not clear before the present studies, and most experimental work addressing parametric dependences of ion heat transport was in fact carried out in rotating plasmas with high NBI heating. In this section we investigate the parametric dependences of ion threshold by using only low rotation plasmas and relying on the observation that at low rotation ions are stiff, so the actual measure of $\mathrm{R} / \mathrm{L}_{\mathrm{Ti}}$ even for on-axis ICRH gives a good approximation of the threshold value. From the theory side, linear stability analysis was carried out with the GS2 code, with Miller equilibrium, electrostatic approximation and collisions, calculating the linear growth rate at different $\mathrm{R} / \mathrm{L}_{\mathrm{Ti}}$ in order to extrapolate the threshold value at

$\mathrm{q}_{\mathrm{i}}{ }^{\mathrm{GB}}=0$. The value of $\mathrm{k}_{\theta} \rho_{\mathrm{i}}=0.3$ was identified for the most unstable mode and used throughout the analysis. For the reference case shown in figure 1a (for which GS2 and FULL yield a linear threshold $\left.\mathrm{R} / \mathrm{L}_{\mathrm{Ti}} \sim 3.6\right)$ a very detailed sensitivity study of the value of linear threshold has been carried out using both GS2 and FULL [43]. It was found that electromagnetic and geometric effects have little effects on the threshold value; even considering up-down asymmetric equilibria with FULL the value is shifted up by only $\Delta \mathrm{R} / \mathrm{L}_{\mathrm{Ti}} \sim 0.5$. Changing $\mathrm{Z}_{\text {eff }}$ or adding a hot deuterium beam species also has little effect. Adding different species (such as impurities or ICRH fast ion minority species) can have a significant effect if the temperature profile of the species is significantly different from the main ion temperature profile. However, in the absence of measurements, such effects have been ignored, and when additional species have been added they have been assumed with the same $\mathrm{R} / \mathrm{L}_{\mathrm{Ti}}$ as the D ions. Finally, whilst the stability analysis at $\rho_{\text {tor }}=0.33$ is in general well defined as ITGs are the only or well dominant instabilities, at $\rho_{\text {tor }}=0.64$ due to the large $\mathrm{R} / \mathrm{L}_{\mathrm{Te}}$ multiple roots are found, which complicate the determination of an ion threshold. Therefore the experimental analysis in the 
next subsections is all carried out at $\rho_{\text {tor }}=0.33$.

\subsection{THE EFFECT OF $T_{E} / T_{I}$ ON ION THRESHOLD}

The role of $\mathrm{T}_{\mathrm{e}} / \mathrm{T}_{\mathrm{i}}$ has been extensively investigated experimentally [44-48] and theoretically $[4,32,49,50]$. Most of this experimental work has been carried out in plasmas with rotation [44-47] and a significant degradation of ion transport with increasing $\mathrm{T}_{\mathrm{e}} / \mathrm{T}_{\mathrm{i}}$ is reported. However in these studies there was no monitoring of rotation changes and their possible concomitant effects. In [48] the need to take into account the concomitant effect of $\mathrm{T}_{\mathrm{e}} / \mathrm{T}_{\mathrm{i}}$ and rotational shear was recognized. However, the two parameters were strongly coupled in the experiments, and the effect of each could not be experimentally separated. An attempt was made to estimate the contribution of the rotational shear using linear GS2 simulations, however assuming a ExB shear threshold up-shift from the Waltz rule, which according to the present JET findings is an underestimate of the rotation effect. The indication emerged anyway that the $\mathrm{T}_{e} / \mathrm{T}_{\mathrm{i}}$ dependence is milder when the rotation contribution is taken into account.

In JET, scans of $\mathrm{T}_{\mathrm{e}} / \mathrm{T}_{\mathrm{i}}$ at similar q profile ( $\mathrm{s} \sim 0.5$ at $\rho_{\text {tor }}=0.33$ ) were carried out at low rotation, using the variation of power deposition from dominant electron in 3\% $(\mathrm{H})$-D minority to dominant ion in $6-8 \%\left({ }^{3} \mathrm{He}\right)-\mathrm{D}$ to dominant electron in $20 \%\left({ }^{3} \mathrm{He}\right)-\mathrm{D}$ where mode conversion takes place. This led to a range in $\mathrm{T}_{\mathrm{e}} / \mathrm{T}_{\mathrm{i}}$ at $\rho_{\text {tor }}=0.33$ from 1 to 1.6. To extend this range, previous experimental JET data in L-mode plasmas with $\mathrm{B}_{\mathrm{T}}=3 \mathrm{~T}, \mathrm{Ip}=1.3 \mathrm{MA}, \mathrm{n}_{\mathrm{e} 0}=210^{19} \mathrm{~m}^{-3}$ and pure electron heating by Lower Hybrid (LH) were also considered, providing an excursion from $\mathrm{T}_{\mathrm{e}} / \mathrm{T}_{\mathrm{i}} \sim 1.7$ to $\sim 3.8$ at $\rho_{\text {tor }}=0.33$, although not being a fully homogeneous data-set with the main one. It is important to note that due to the very high ion stiffness at low rotation as discussed above, it is experimentally impossible to produce low rotation plasmas with $\mathrm{T}_{\mathrm{e}} / \mathrm{T}_{\mathrm{i}}<1$, since adding ion power does not increase $\mathrm{R} / \mathrm{L}_{\mathrm{Ti}}$ and on the other hand the mere Ohmic power is sufficient to heat electrons at a similar level given their much lower stiffness level.

The results are summarized in figure 9. Red full circles are the measured $\mathrm{R} / \mathrm{L}_{\mathrm{Ti}}$ at $\rho_{\text {tor }}=0.33$ of low rotation discharges and indicate a variation $\Delta \mathrm{R} / \mathrm{L}_{\mathrm{Ti}} \sim 1$ for $1<\mathrm{T}_{\mathrm{e}} / \mathrm{T}_{\mathrm{i}}<1.6$. The dependence from the second data-set in the range $1.7<\mathrm{T}_{\mathrm{e}} / \mathrm{T}_{\mathrm{i}}<3.8$ seems instead weaker. For comparison, in some discharges NBI power has been added, inducing rotation at two different levels. In these plasmas due to dominant NBI heating $\mathrm{T}_{\mathrm{e}} / \mathrm{T}_{\mathrm{i}} \leq 1$ and it is not possible to increase it because electrons become stiff and ion non-stiff, so the problem is reversed. It is evident that $\mathrm{R} / \mathrm{L}_{\mathrm{Ti}}$ exceeds threshold by a large factor as discussed previously, and dependences of stiffness on rotation and s completely mask the $\mathrm{T}_{\mathrm{e}} / \mathrm{T}_{\mathrm{i}}$ dependence of threshold. At low rotation, $\mathrm{R} / \mathrm{L}_{\mathrm{Ti}}$ is already a good estimate of threshold due to high stiffness, however an even better estimate can be achieved by assuming that the same stiffness level measured for $\mathrm{T}_{\mathrm{e}} / \mathrm{T}_{\mathrm{i}}=1$ holds at all values of $\mathrm{T}_{\mathrm{e}} / \mathrm{T}_{\mathrm{i}}$ and then extrapolating to $\mathrm{q}_{\mathrm{i}}{ }^{\mathrm{GB}}=0$, as shown in figure 9b. This procedure yields the red open circles in figure 9a. For the ICRH dataset linear GS2 simulations were carried out, whose results for a $\mathrm{T}_{\mathrm{e}} / \mathrm{T}_{\mathrm{i}}$ scan with $\mathrm{s} / \mathrm{q}=0.57 / 1.3$ ( = 0.44 ) are shown in figure 9a. The agreement between data and GS2 predictions is pretty good in the range of $\mathrm{T}_{\mathrm{e}} / \mathrm{T}_{\mathrm{i}}$ explored. Eq.(2), although being a simplified model, can be used to infer the 
trend over a broader range and compare with the $\mathrm{LH}$ data-set. Here the range $0.23<\mathrm{s} / \mathrm{q}<0.44$ has been used because for the LH data-set MSE measurements are not available, so the q profile is somewhat uncertain. There is qualitative agreement with data in the prediction of a weaker trend at higher $\mathrm{T}_{\mathrm{e}} / \mathrm{T}_{\mathrm{i}}$. As mentioned above, the stronger dependence predicted for $\mathrm{T}_{\mathrm{e}} / \mathrm{T}_{\mathrm{i}}<1$ cannot be explored experimentally in absence of rotation. The conclusion is that in the core plasma where $\mathrm{s} / \mathrm{q}<0.6$ the effect of $\mathrm{T}_{\mathrm{e}} / \mathrm{T}_{\mathrm{i}}$ on ion threshold is modest in the range $\mathrm{T}_{\mathrm{e}} / \mathrm{T}_{\mathrm{i}}>1$ (which is the relevant range for ITER operations). Large effects previously observed in rotating plasmas may be due both to concomitant rotation changes affecting ion stiffness and a stronger effect of $\mathrm{T}_{\mathrm{e}} / \mathrm{T}_{\mathrm{i}}$ on threshold in the range $T_{e} / T_{i}<1$. In the outer part of the plasma where s/q is higher a stronger $T_{e} / T_{i}$ dependence is theoretically expected (see Eq.(2)), however in that region it is rather difficult to achieve $\mathrm{T}_{\mathrm{e}} / \mathrm{T}_{\mathrm{i}}$ values significantly departing from 1.

\subsection{THE EFFECT OF S/Q ON ION THRESHOLD}

The low rotation part of the experiments shown in figure 1c (made to study the effect of $s$ on ion stiffness mitigation) constitutes an ideal dataset to study the impact of s/q on ion threshold. They are all characterized by $\mathrm{T}_{\mathrm{e}} / \mathrm{T}_{\mathrm{i}} \sim 1$. Figure 10 shows that $\mathrm{R} / \mathrm{L}_{\mathrm{Ti}}$ at low rotation (a good approximation of threshold due to high stiffness) has a very weak positive dependence on s/q. The high rotation shots, shown for comparison, have much higher $\mathrm{R} / \mathrm{L}_{\mathrm{Ti}}$ due to stiffness reduction, decreasing with s as discussed above (see also figure 6b). The predictions of Eq.(2) are also shown, indicating reasonable agreement with the low rotation data. Linear gyro-kinetic thresholds have been calculated using GS2 when varying s and q separately. It turns out that in this region of parameters it is q rather than $s$ that impacts on threshold, so that the two curves are not identical. In any case, the dependence of threshold on q profile is extremely weak for these shots in the core region, both in experiment and theory.

\section{CONCLUSIONS}

This paper has presented first detailed ion heat transport studies in JET in which heat flux scans and $T_{i}$ modulation have allowed to separately identify ITG threshold and stiffness and study some parametric dependences. Threshold was found to behave as expected and also in good quantitative match with linear gyro-kinetic predictions, although no evidence of a non-linear threshold upshift emerges from the data, whilst it is present in non-linear gyro-kinetic simulations. The main mismatch between experiment and theory however regards the behaviour of stiffness. In experiments ion stiffness is extremely high in non-rotating plasmas, so that the $\mathrm{T}_{\mathrm{i}}$ profile is dominated by threshold behaviour. However, stiffness is dramatically reduced when rotational shear is high and magnetic shear is low, and in these conditions the $\mathrm{T}_{\mathrm{i}}$ profile is completely dominated by stiffness, which opens the possibility of improved ion core confinement. Theory yields mixed results on the stiffness reduction with rotation and low magnetic shear, with the more refined non-linear gyro-kinetic models being more distant from experiment than the quasi-linear fluid/gyro-fluid models. In addition, too high $\mathrm{R} / \mathrm{L}_{\mathrm{Ti}}$ values are predicted at low rotation by all models, either due 
to not high enough stiffness or to the presence of a Dimits threshold up-shift. Global simulations did not help to improve such discrepancies with respect to experiment. Solving these problems appears a necessary step if we want to have a validated tool for predictions of ion heat transport in next generation devices. Although in JET the experimental result is robust and well reproducible, before claiming that there is something missing in gyro-kinetic simulations, one should first seek similar experimental evidence in machines different from JET, in order to provide further support to the experimental result. An ITPA joint project is already in place on this topic and experiments are being performed on C-MOD, DIII-D, AUG, TEXTOR and MAST. On other hand, more nonlinear gyro-kinetic simulations should be performed, comparing global vs local runs and fixedgradient vs fixed-flux approaches. One issue is that these simulations should address the region near marginality, which is the most challenging numerically and requires well-resolved, long and expensive simulations, but is in fact the operating domain of fusion relevant devices. Whilst waiting for further developments of the work both on the experimental and theoretical fronts, some indications emerge from the JET experiments in view of ITER operations:

- in standard H-mode, the operation in absence of a significant rotational shear will not affect ITER performance much, since at high s the effect of stiffness mitigation is seen small. In these conditions, the most reliable predictions are the ones by stiff quasi-linear models (such as TGLF, GLF23, Weiland) although they are still possibly on the optimistic side in conditions well above threshold, as stiffness may be even higher. The non-linearly increased $\mathrm{R} / \mathrm{L}_{\mathrm{Ti}}$ yielded by non-linear gyro-kinetic simulations does not find support in present data. This conclusion is on the pessimistic side, since having core $\mathrm{R} / \mathrm{L}_{\mathrm{Ti}} \sim 4$ places a major role on the pedestal height in order to achieve $\mathrm{Q}=10$, with all related problems for plasma-wall interaction.

- A dramatic effect of the absence of rotational shear is instead envisaged for ITER AT scenarios, which require improved core ion confinement and therefore would not be achievable without the beneficial effect of rotational shear. This argument can however be turned into a very positive one, if as it seems from recent momentum transport studies [5153 ] there will likely be rotation peaking even in the absence of core torque sources due to a sizeable momentum pinch. In fact, the beneficial effect of rotational shear through stiffness mitigation is seen in JET to exceed by far the well-known beneficial effect due to ExB shear threshold up-shift, thereby allowing operation with much higher $\mathrm{R} / \mathrm{L}_{\mathrm{Ti}}$ than presently foreseen and so with lower pedestal. In order to achieve these conditions, however, the possibility of operation with a core region of low magnetic shear must be guaranteed.

\section{ACKNOWLEDGMENT}

Nonlinear gyro-kinetic simulations were performed on the parallel server Power 6 (Vip) of the IPPMPG Rechenzentrum Garching, Germany. This work, supported by the European Communities under the contract of Association EURATOM/ENEA-CNR, was carried out within EFDA. The views and opinions expressed herein do not necessarily reflect those of the European Commission. 


\section{REFERENCES}

[1]. Mattor N et al 1988 Physics of Fluids 311180

[2]. Romanelli F et al 1989 Physics of Fluids B 11018

[3]. Connor JW and Wilson HR 1994 Plasma Physics and Controlled Fusion 36719

[4]. Kotschenreuther M et al 1995 Physics of Fluids 22381

[5]. Special Issue on Joint European Torus (JET), Fusion Science and Technology 53, No.4 (2008)

[6]. Giroud C et al 2008 Review of Scientific Instruments 79 10F525

[7]. Van Eester D et al 2009 Plasma Physics and Controlled Fusion 51044007

[8]. Ryter F et al 2006 Plasma Physics and Controlled Fusion 48 B453

[9]. Mantica P and Ryter F 2006 C.R. Physique 7634

[10]. Waltz R.E. et al 1994 Physics of Plasmas 12229

[11]. Dimits AM et al 2000 Physics of Plasmas 7969

[12]. Mantica P et al 2009 Physical Review Letters 102175002

[13]. Ryter F et al 2011 "Simultaneous Analysis of Ion and Electron Heat Transport by Power Modulation in JET", submitted to Nuclear Fusion

[14]. Garbet X et al 2004 Plasma Physics and Controlled Fusion 461351

[15]. Hedin J et al 2002 Nuclear Fusion 42527

[16]. Eriksson LG et al 1993 Nuclear Fusion 331037

[17]. Challis CD et al 1989 Nuclear Fusion 29563

[18]. Mantica P et al 2011 submitted to Physics Review Letters

[19]. Lopes Cardozo NJ et al1997 Plasma Physics and Controlled Fusion 39 B303

[20]. Austin ME et al 2006 Physics of Plasmas 13082502

[21]. Joffrin E et al 2002 Nuclear Fusion 42235

[22]. de Vries PC et al 2009 Nuclear Fusion 49075007

[23]. Waltz RE et al 2006 Physics of Plasmas 13052301

[24]. Figueiredo ACA et al 2008 Review of Scientific Instruments $7910 \mathrm{~F} 107$

[25]. Figueiredo ACA et al 2009 36th EPS Conf. on Plasma Physics, Sofia, 2009 ECA Vol.33E, P-2.167

[26]. Baiocchi B et al in preparation

[27]. Tala T et al. 2011 Plasma Physics and Controlled Fusion 43506

[28]. Frassinetti L et al 2010 37th EPS Conference on Plasma Phys. Dublin, 2010, P-1.1031

[29]. de Vries PC et al 2008 Plasma Physics and Controlled Fusion 50065008

[30]. Politzer PA et al 2008 Nuclear Fusion 48075001

[31]. Kotschenreuther M et al 1995 Computational Physics Communication 88128

[32]. Guo SC and Romanelli F 1993 Physics of Fluids B 5520

[33]. Versloot TW et al 2011 submitted to Nuclear Fusion

[34]. Weiland J 2000 Collective Modes in Inhomogeneous Plasmas (IOP, Bristol, 2000)

[35]. Waltz RE et al 1997 Physics of Plasmas 42482 
[36]. Staebler GM et al 2005 Physics of Plasmas 12102508

[37]. Weiland J and Mantica P 2011 38th EPS Conference on Plasma physics, Strasbourg, P-xxx

[38]. Candy J and Waltz RE 2003 journal Computational Physics 186545

[39]. Peeters AG et al 2009 Computational Physics Communication 1802650

[40]. Mantica P et al 2010 23rd IAEA Fusion Energy Conference, Daejeon, 2010, (IAEA, Vienna) EXC/9-2

[41]. Highcock EG et al 2010 Physical Review Letters 105215003

[42]. Sugita S et al 2010 37th EPS Conference on Plasma Phys. Dublin, 2010 P1.1095

[43]. Rewoldt G et al 1982 Physics of Fluids 25480

[44]. Petty CC et al 1999 Physical Review Letters 833661

[45]. Suttrop W et al 2001 Europhys. Conf. Abstract A 25989

[46]. Asp E et al 2005 Plasma Physics and Controlled Fusion 47505

[47]. Manini A et al 2004 Plasma Physics and Controlled Fusion 461723

[48]. Manini A et al 2006 Nuclear Fusion 461047

[49]. Weiland Jet al 2005 Plasma Physics and Controlled Fusion 47441

[50]. Casati A et al 2008 Physics of Plasmas 15042310

[51]. Mantica P et al 2010 Physics of Plasmas 17092505

[52]. Tala T et al 2011 submitted to Nuclear Fusion

[53]. Solomon WM et al 2009 Nuclear Fusion 49085005 
(a)
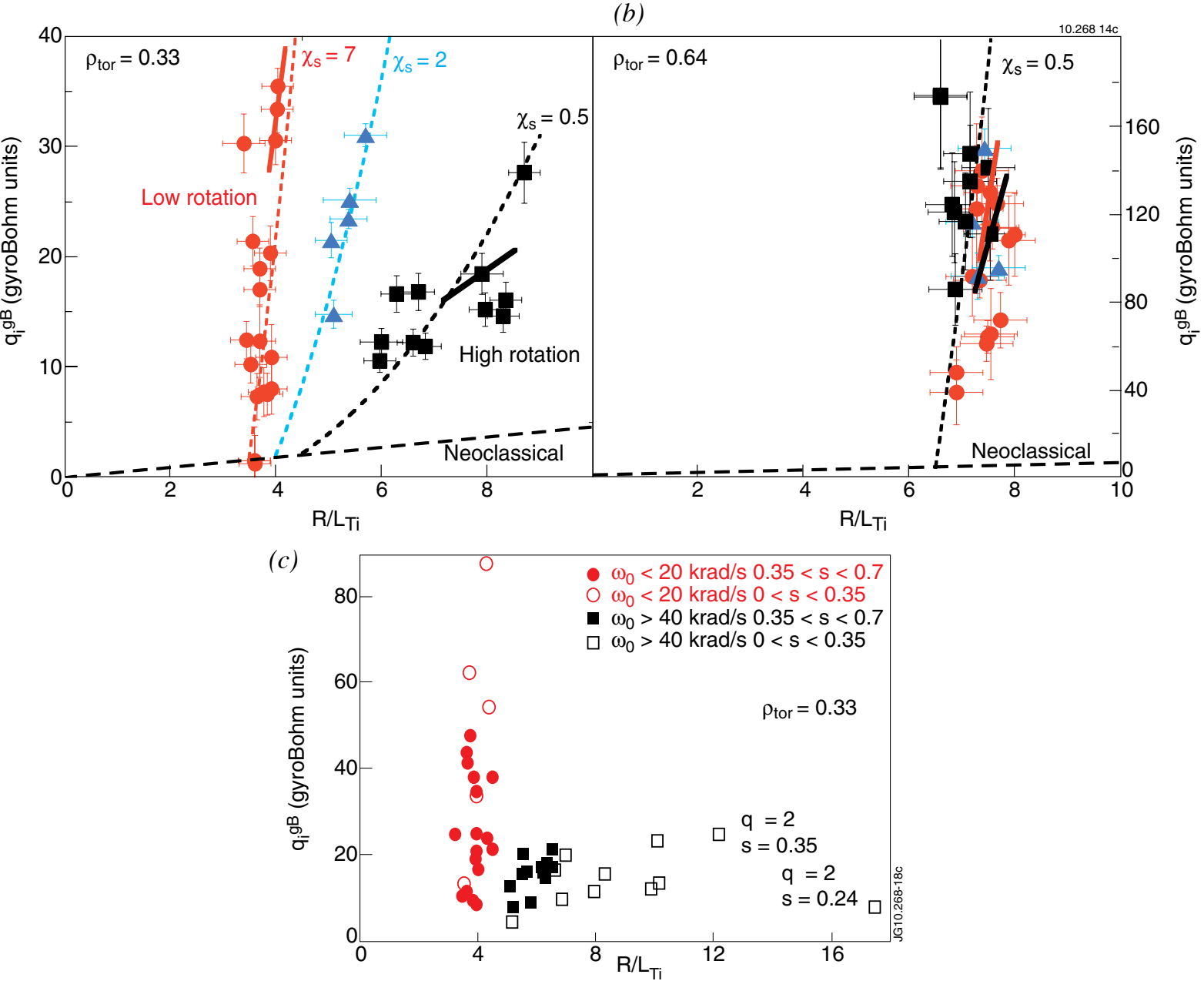

Figure 1: $q_{i}^{G B}\left[=q_{i} /\left[\left(\rho_{i} / R\right) 2 v_{i t h} n_{i} T_{i}\right]\right.$ with $\left.v_{i t h}=\left(T_{i} / m_{i}\right) 1 / 2, \rho_{i}=\left(T_{i} m_{i}\right)^{1 / 2} / e B\right]$ vs $R / L_{T i}$ at $(a) \rho_{\text {tor }}=0.33,(b) \rho_{\text {tor }}=0.64$ for similar plasmas with different levels of rotation. $\bullet: 1<\omega_{t 0}<210^{4} \mathrm{rad} / \mathrm{s}, \Delta: 3<\omega_{t 0}<410^{4} \mathrm{rad} / \mathrm{s}, \mathbf{m}: 5<\omega_{t 0}<610^{4} \mathrm{rad} / \mathrm{s}$. The dashed black line is indicative of neoclassical transport. The segments indicate the local slope deduced from modulation. The dotted lines represent the Critical Gradient Model (CGM) [11] with different values of cs. c) Same at $\rho_{\text {tor }}=0.33$ for similar plasmas with different rotation and $s$ values.

(After [18], copyright 2011 American Physical Society). 
(a)

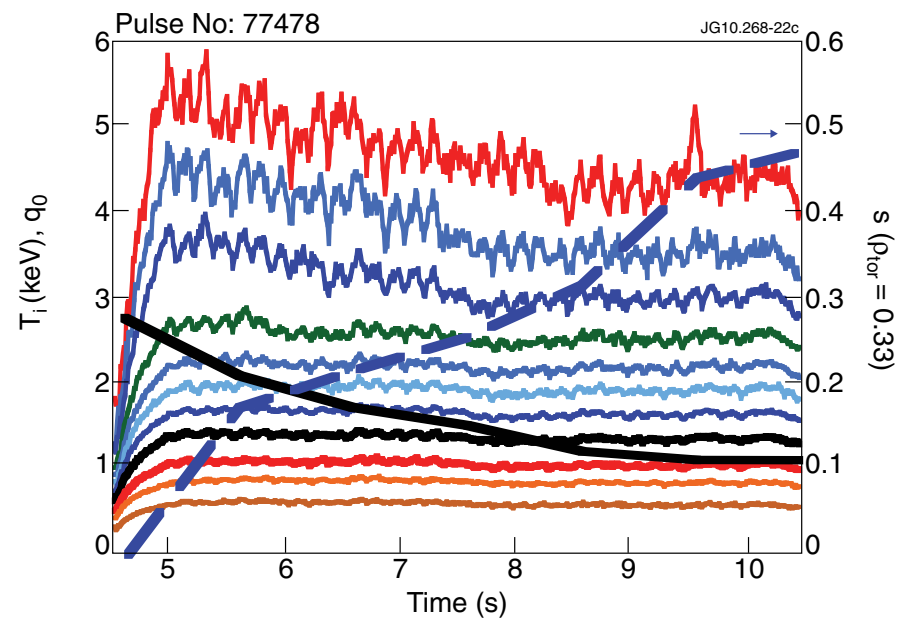

(b)

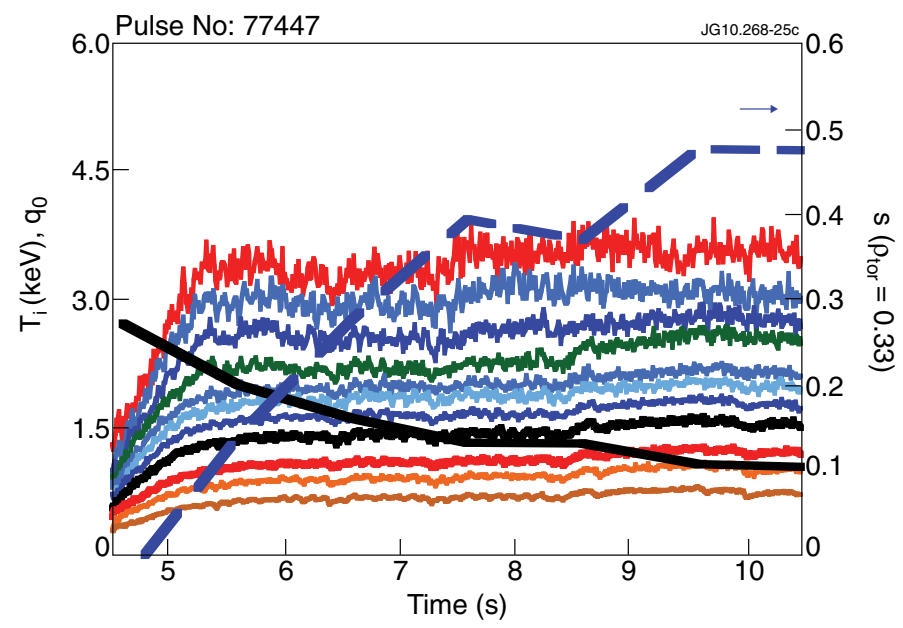

(c)

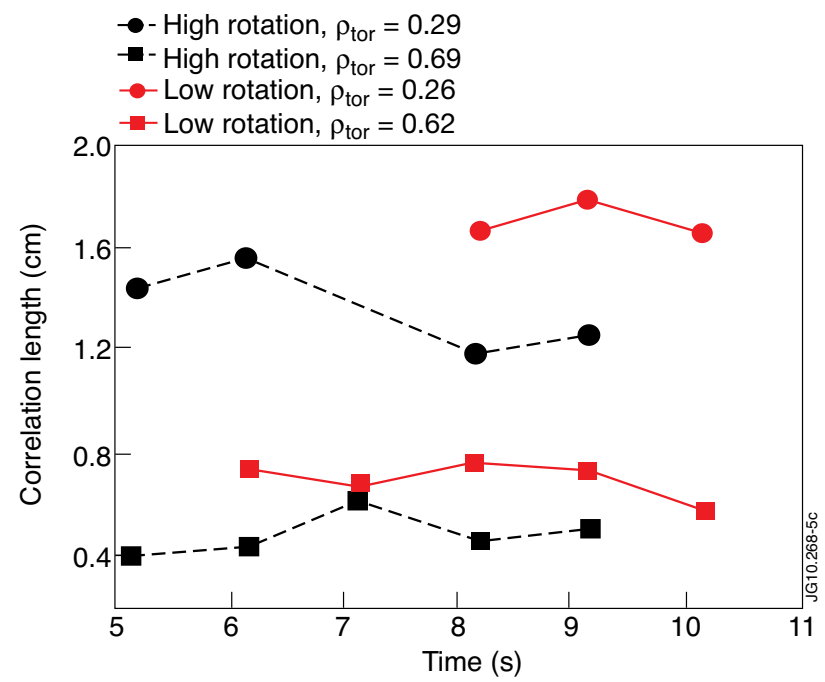

Figure 2: a) Experimental (modulated) $T_{i}$ at different radii versus time for the high rotation Pulse No: 77478 and $b$ ) for the low rotation Pulse No: 77447. The thick full line is the central $q$ and the thick dashed line is $s$ at $\rho_{\text {tor }}=0.33$. c) Time evolution of the reflectometer radial correlation length at two locations for a low (Pulse No: 77455) and a high (Pulse No: 77477 ) rotating shot. 

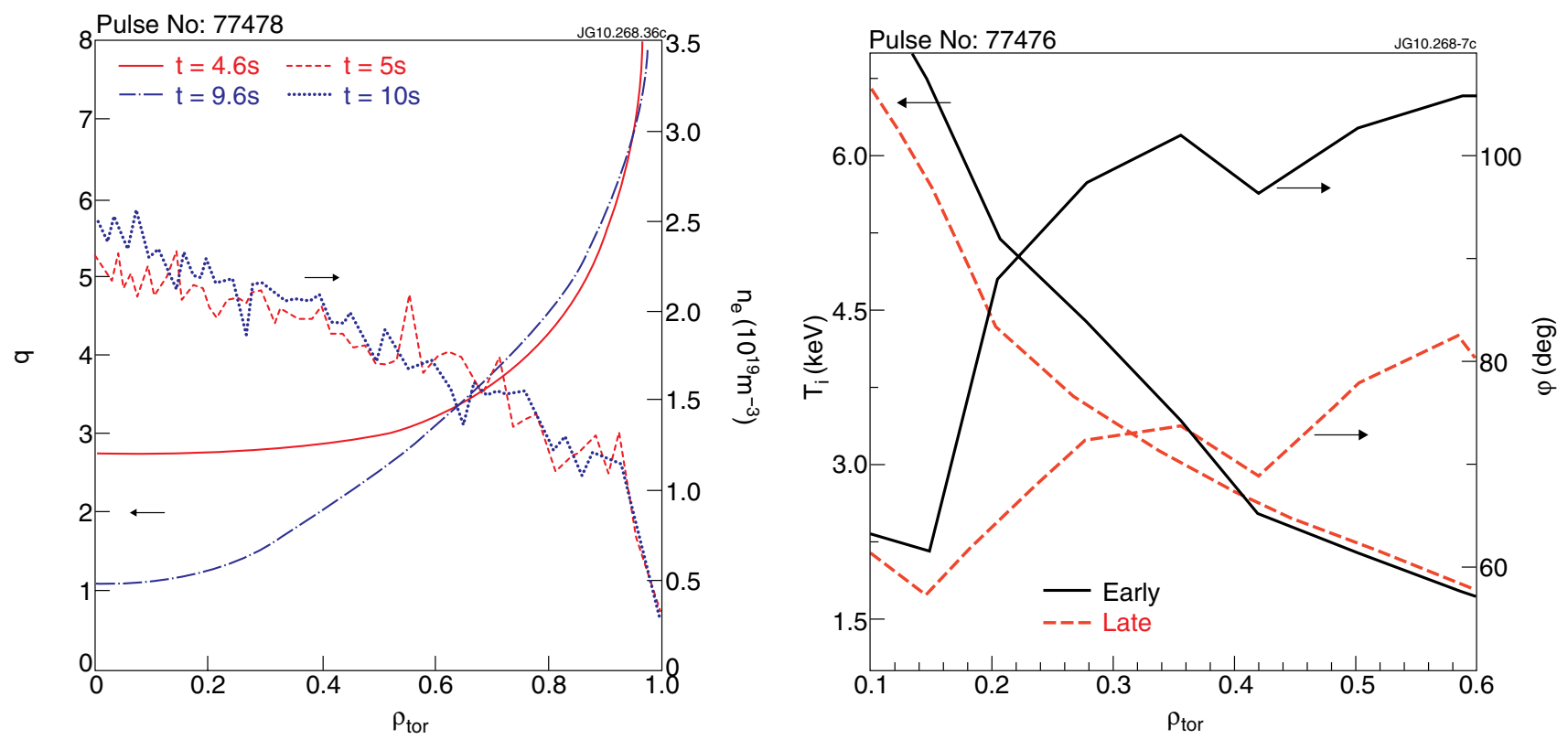

Figure 3: a) Profiles of q from MSE and ne from HRTS at early and late times for the high rotation discharge 77478. The late ne profile has been renormalized to match the edge value at early time. b) Profiles of Ti and Ti modulation phase at early and late times for the high rotation discharge 77476.
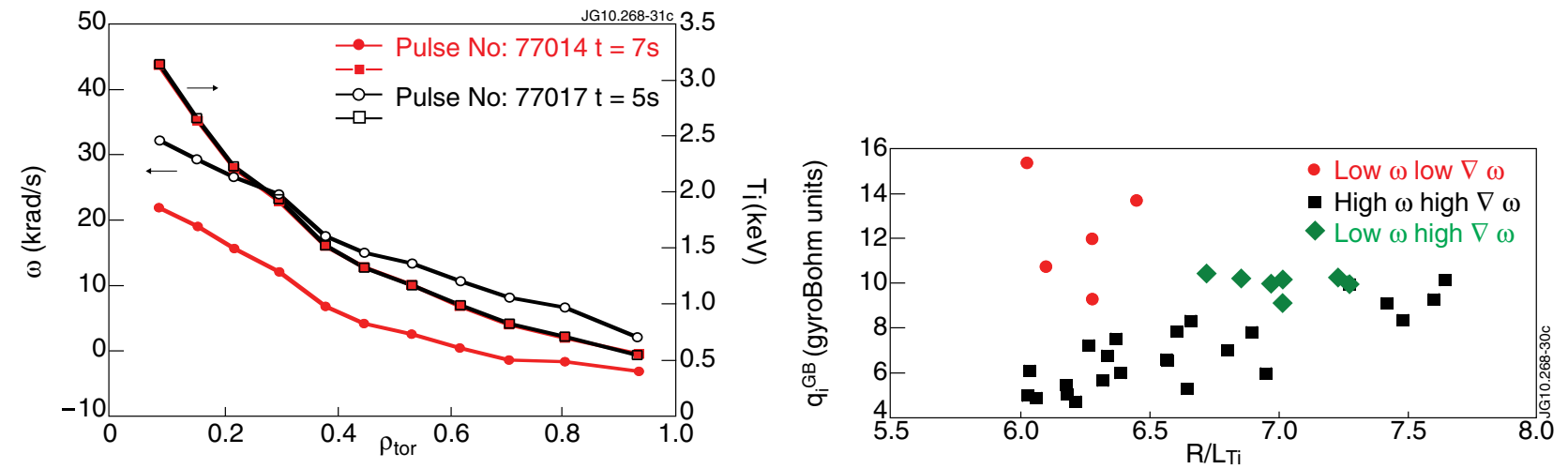

Figure 4: a) rotation (circles) and Ti (squares) profiles with standard (open symbols) and enhanced (full symbols) BT ripple b) $q_{i}^{G B}$ versus $R / L_{T i}$ at $\rho_{\text {tor }}=0.25$ in discharges where $\omega$ and $\nabla \omega$ were decoupled using enhanced BT ripple. 


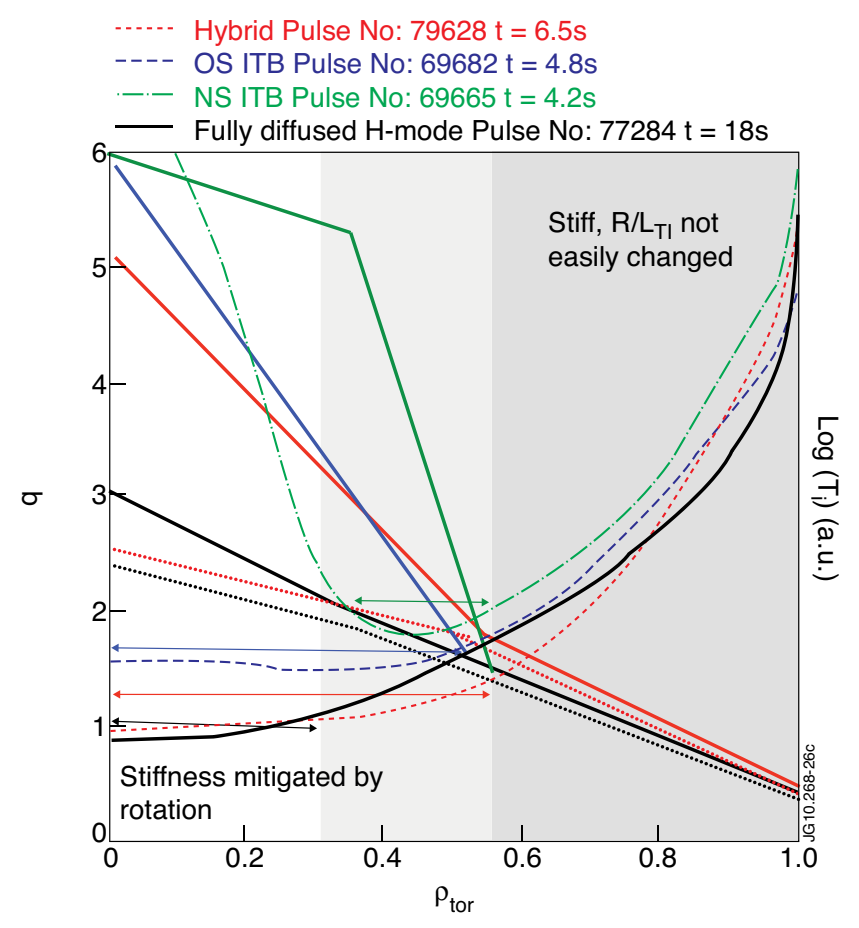

Figure 5: Sketch for core ion stiffness mitigation in Hybrid and Ion ITBs (Optimized or Negative magnetic Shear). qand $\log T_{i}$ profiles are shown, arrows indicate the spatial extent of the low s region. See detailed explanation in main text.

(a)

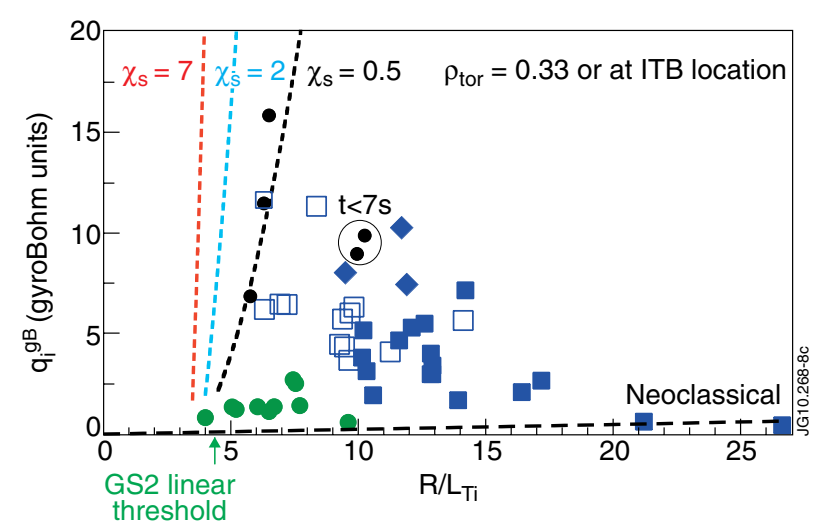

(b)

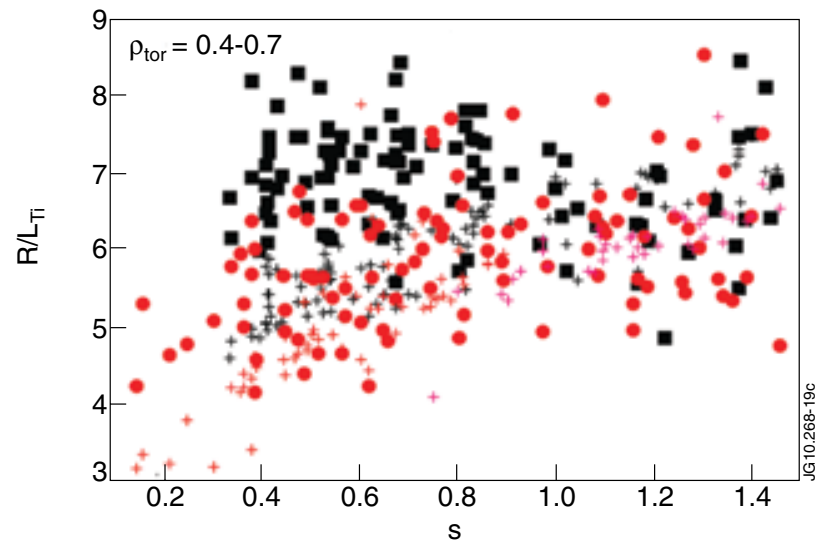

Figure 6: a) $q_{i}^{G B}$ versus $R / L_{T i}$ at $\rho_{\text {tor }}=0.33$ for a set of Hybrids (green circles), ion ITBs at trigger time (blue open squares) and fully developed (blue full squares, diamonds with large ICRH fraction and reduced rotation). Fig1 a data is indicated by CGM fits. Neoclassical level and GS2 linear threshold for an Hybrid are shown. $b$ ) $R / L_{T i}$ versus $s$ at various locations for $\rho_{\text {tor }}=0.4-0.7$ at low (red circles, $\nabla \omega_{t}<50 \mathrm{krad} /(\mathrm{ms})$ ) and high (black squares, $\nabla \omega_{t}>130 \mathrm{krad} /$ (ms))) rotation from a Hybrid and H-mode JET database. Crosses are threshold values from Eq.(2). (Adapted from [18], copyright 2011 American Physical Society) 

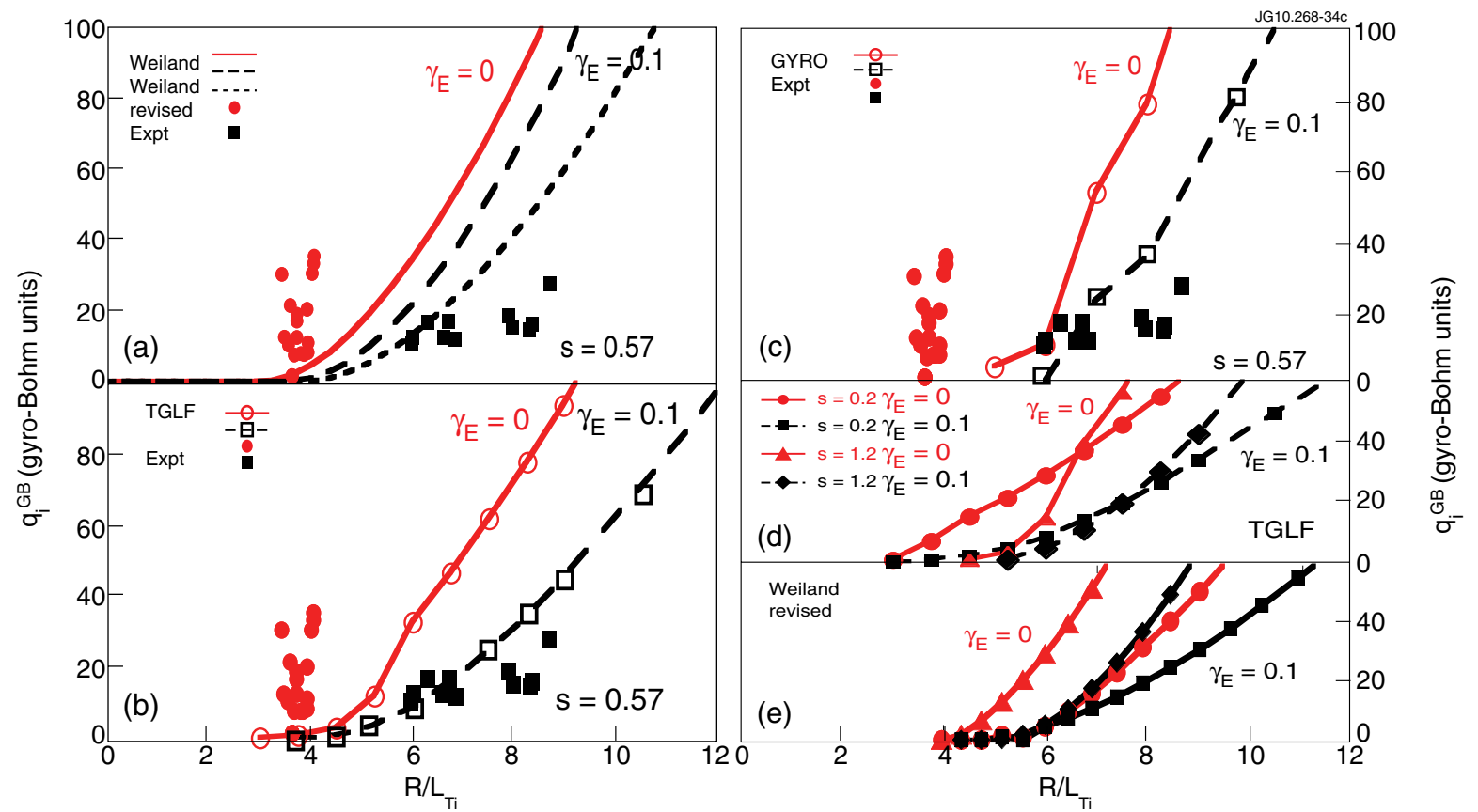

Figure 7: $q_{i}^{G B}$ versus $R / L_{T i}$ at $\rho_{\text {tor }}=0.33$ without rotation and with $\gamma_{E} \sim 0.1$ from a set of a) Weiland, $\left.b\right)$ TGLF and c) GYRO simulations with $s=0.57$ compared with the data of Fig.la.d) TGLF and e) revised Weiland (legend as in d)) simulations where s has been set to 0.2 and 1.2 but otherwise identical to those in a), b), c). (After [18], copyright 2011 American Physical Society)

(a)

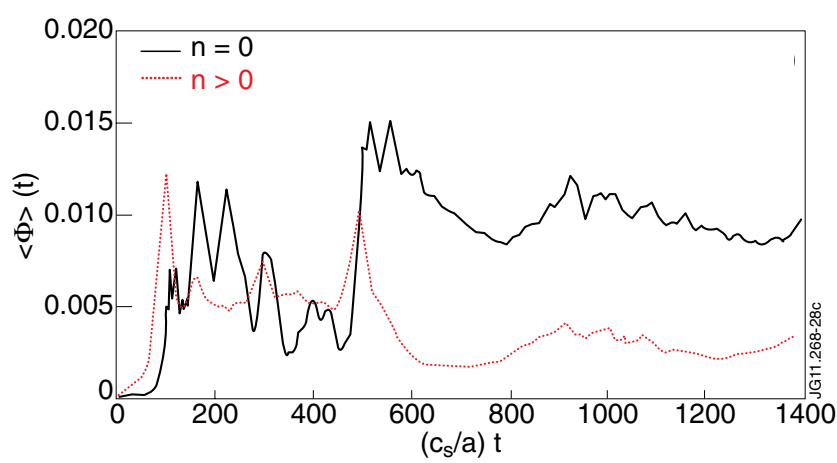

(b)

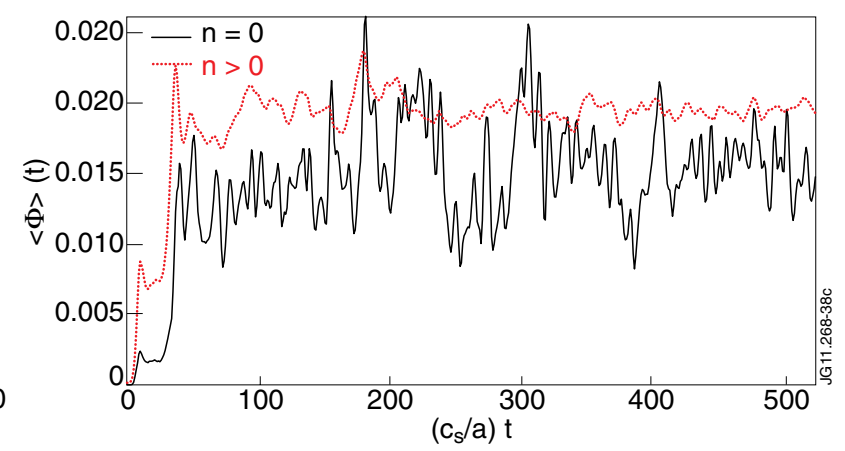

Figure 8: Time evolution of RMS electrostatic potential distinguishing $n=0$ (zonal) and $n>0$ com-ponents in a nonlinear flux-tube GYRO simulations with 64 toroidal modes, $\gamma_{E}=0$ and a) $\left.R / L_{T i}=5, b\right) R / L_{T i}=10$. 
(a)

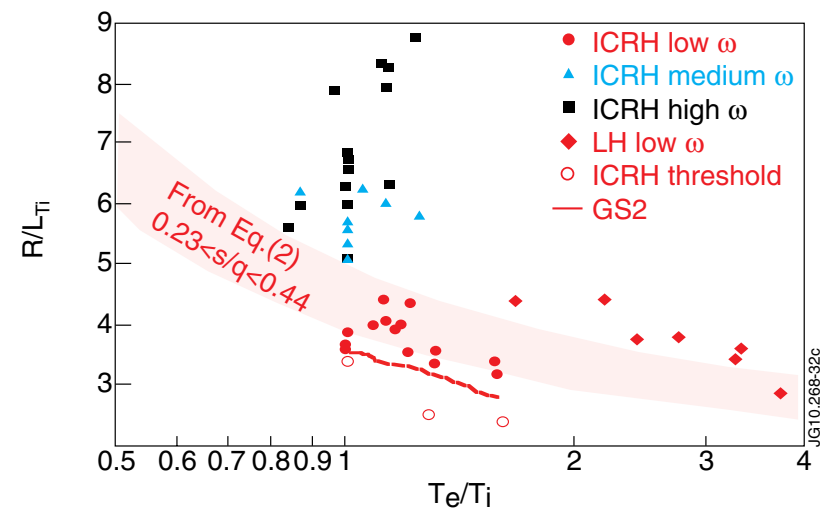

(b)

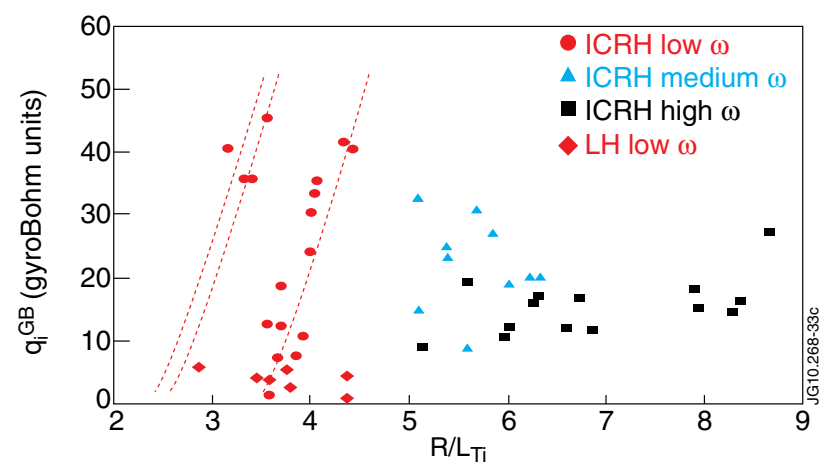

Figure 9: a) $R / L_{T i}$ versus $T_{e} / T_{i}$ at $\rho_{\text {tor }}=0.33$ at different rotation levels (indicated as in Fig.1a). Full circles are actual $R /$ LTi and open circles are extrapolations to $q_{i}^{G B}=0$ as indicated in b). The dotted band is after Eq.(2) for $0.23<s /$ $q<0.44$ and the line is the GS2 linear threshold at $s / q=0.57 / 1.3 . b) q_{i}^{G B}$ versus $R / L_{T i}$ at $\rho_{\text {tor }}=0.33$ for the same shots as in a). The lines are CGM fits of the $T_{e} / T_{i}=1$ data, translated in R/LTi in order to deduce the threshold values at different $T_{e} / T_{i}$ for the ICRH discharges.

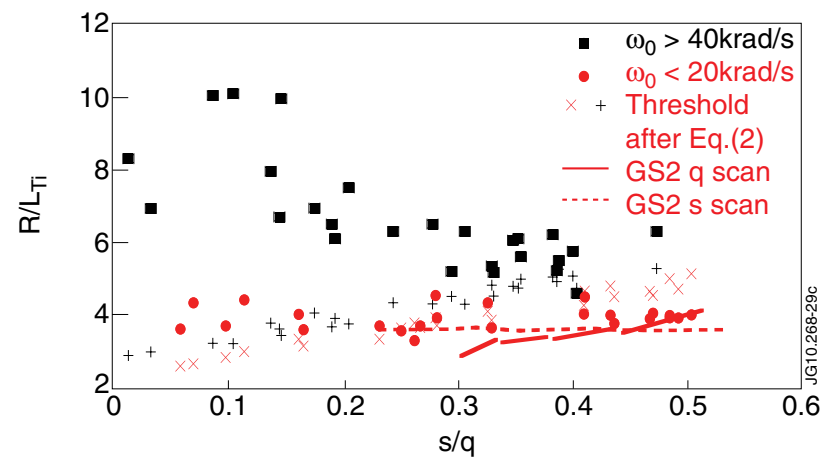

Figure 10: $R / L_{T i}$ versus $T_{e} / T_{i}$ at $\rho_{\text {tor }}=0.33$ at low and high rotation $\left(T_{e} / T_{i} \sim 1\right)$ ( same data as figure $\left.1 c\right)$. Crosses are threshold values after Eq.(2) and lines are GS2 linear thresholds for a $q$ and a s scan. 